\title{
Factores asociados con la lactancia materna exclusiva en el Perú: análisis de la Endes 2017
}

\author{
Factors associated with exclusive breastfeeding in Peru: \\ Analysis of Endes 2017
}

\author{
Rodrigo Daga ${ }^{10}$ \\ rodrigo.dagasoto@gmail.com
}

Artículo recibido: 23/05/2021

Revisado por pares

Artículo aceptado: 25/01/2022

Artículo publicado: 25/02/2022

Autor de correspondencia

Rodrigo Daga,

rodrigo.dagasoto@gmail.com

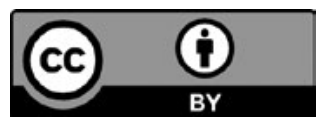

(CEl autor, 2022. Publicado por la Universidad Norbert Wiener (Lima, Perú)

Citar como: Daga R. Factores asociados a la lactancia materna exclusiva en el Perú: análisis de la Endes 2017. Revista de Investigación (de la Universidad Norbert Wiener). 2022; 11(1): a0005. doi: https://doi.org/10.37768/unw.rinv.11.01.a0005

\begin{abstract}
Resumen
Introducción: La lactancia materna exclusiva es el método adecuado para proporcionar nutrientes e inmunoglobulinas al infante durante los seis primeros meses de vida. En diversas regiones se han implementado políticas que promueven la lactancia materna exclusiva para prevenir la desnutrición calórica y proteica infantil. Sin embargo, a pesar de los esfuerzos de los sistemas de salud, instituciones públicas y privadas, la frecuencia de lactancia materna ha disminuido mundialmente según los últimos informes internacionales. Objetivo: Identificar los factores asociados con la lactancia materna exclusiva en el Perú, durante el año 2017. Material y métodos: Se realizó un análisis descriptivo, y multivariado de los datos de la Endes 2017, considerando el diseño muestral. En el análisis multivariado, se utilizaron modelos de regresión de Poisson múltiple con las variables con significancia estadística en los modelos regresión de Poisson simple. Resultados: La prevalencia de la práctica de lactancia materna exclusiva en el Perú fue del $63,82 \%$ en los infantes menores de 6 meses. Se identificó que el índice de riqueza y el sexo al nacer al infante se asociaron con la práctica de lactancia materna exclusiva en infantes menores de 6 meses. Conclusiones: Las madres con ingresos económicos bajos tuvieron mayor probabilidad de prácticar la lactancia materna exclusiva durante los 6 primeros meses de vida del infante, en comparación con las madres de ingresos económicos intermedios, intermedios-altos y altos. Asimismo, los infantes de sexo femenino tuvieron mayores probabilidades de recibir lactancia materna exclusiva que los infantes de sexo masculino.
\end{abstract}

Palabras clave: lactancia materna exclusiva, determinantes sociales de la salud, fenómenos fisiológicos nutricionales de la infancia

\begin{abstract}
Introduction: Exclusive breastfeeding is the appropriate method to provide nutrients, and immunoglobulins to infants during the first six months of life. In several regions, policies have been implemented to promote exclusive breastfeeding to prevent infant protein- energy malnutrition. However, despite the efforts of health systems, public and private institutions, the frequency of breastfeeding has decreased worldwide according to the latest international reports. Objective: To identify the factors associated with exclusive breastfeeding in Peru, during 2017. Material and methods: We performed a descriptive, and multivariate analysis of the Endes 2017 data, considering the sample design. In the multivariate analysis, we used multiple Poisson regression models with the variables with statistical significance in the simple
\end{abstract}

${ }^{1}$ Escuela de Nutrición Humana, Universidad Nacional de Educación Enrique Guzmán y Valle. Chosica, Perú. 
Poisson regression models. Results: The prevalence of exclusive breastfeeding in Peru was $63.82 \%$ in infants under six months of age. We identified that wealth index and sex at birth were associated with exclusive breastfeeding practice in infants under six months old. Conclusions: Mothers with low economic income were more likely to practice exclusive breastfeeding during the infant's first six months of life compared to mothers with intermediate, upper-intermediate, and high economic income. Likewise, female infants were more likely to be exclusively breastfed than male infants.

Keywords: exclusive breast feeding, social determinants of health, child nutritional physiological phenomena

\section{INTRODUCCIÓN}

La leche materna es un alimento ideal para el infante durante los primeros meses de vida, brinda nutrientes y anticuerpos esenciales desde la primera eyección; asimismo, no genera costos a la madre y es de origen natural $\left(^{1}\right)$. La lactancia materna exclusiva es una estrategia de salud pública que busca garantizar la adecuada alimentación y nutrición infantil, así como disminuir el riesgo de mortalidad y morbilidad infantil $\left({ }^{2}\right)$.

Algunos estudios han reportado la disminución de la lactancia materna exclusiva en diferentes regiones del mundo, tanto en países desarrollados como en vía de desarrollo $\left({ }^{3,4}\right)$. Se han explorado diferentes factores que influyen en la lactancia materna exclusiva, como los demográficos (zona de residencia), socioeconómicos (nivel de ingresos, nivel educativo), sanitarios (acceso a la salud, tipo de parto) e individuales (preferencias, paridad, planificación del embarazo) $\left(^{3,5-8}\right)$. Sin embargo, la influencia de cada factor puede ser relativa según país y región geográfica, e inclusive en el tiempo.

En diversos países del mundo se han implementado políticas que promueven la lactancia materna exclusiva para prevenir la desnutrición calórica y proteica de los infantes $\left({ }^{9}\right)$. Sin embargo, a pesar de los esfuerzos para promover la lactancia materna mediante los sistemas de salud, instituciones públicas y privadas, esta práctica ha disminuido mundialmente, según los últimos informes internacionales $\left({ }^{10}\right)$. Brindar lactancia materna proporciona beneficios para la madre y el infante, por ejemplo, se asocia con la disminución del riesgo de desnutrición crónica, anemia e infecciones gastrointestinales en el infante, así como la disminución del riesgo de cáncer de cuello uterino y de mama. También disminuye el riesgo de depresión posparto en las madres $\left({ }^{11}\right)$.

Pero la práctica de la lactancia materna no solo tiene efectos durante la infancia, también los tiene en la adolescencia del individuo, al disminuir el riesgo de desarrollar de diabetes mellitus, alergias alimentarias, enfermedades cardiovasculares y obesidad $\left({ }^{12}\right)$.

El conocimiento de los beneficios de brindar lactancia materna no es suficiente en la actualidad, pues aún existen problemas que influyen en la decisión de la madre de amamantar. Algunos que se han reportado son la influencia de la industria, que distribuye sucedáneos de leche materna $\left({ }^{13}\right)$; el ejercicio de actividades laborales $\left({ }^{14}\right)$; el nivel educativo de la madre y el apoyo familiar $\left({ }^{15}\right)$; las características psicológicas del padre y la madre del recién nacido $\left({ }^{16}\right)$, y la participación en actividades de promoción de la lactancia materna.

En el Perú, mediante la Encuesta Demográfica y de Salud Familiar (Endes), que es realizada por el Instituto Nacional de Estadística e Informática (INEI), se recoge información sobre demografía, salud y otros determinantes de salud de la población peruana, para evaluar y mejorar las estrategias sanitarias y políticas sectoriales $\left({ }^{17}\right)$. Los informes de cada Endes presentan resultados sobre la lactancia y nutrición de niñas, niños y mujeres, y presentan estadísticas sobre la lactancia materna inicial y su continuidad durante los seis primeros meses de vida.

Es necesario destacar que, en la población peruana, se han desarrollado investigaciones sobre la asociación entre la lactancia materna exclusiva y algunas características clínicas, socioculturales y demográficas; sin embargo, sus metodologías no 
son tan robustas para realizar inferencias a nivel nacional o por regiones $\left({ }^{18-23}\right)$. Por esa razón, el presente estudio tuvo como objetivo identificar los factores asociados con la lactancia materna exclusiva en el Perú en infantes menores de 6 meses, mediante el análisis de la base de datos de la Endes 2017.

\section{MATERIAL Y MÉTODO}

Se realizó un análisis de las fuentes secundarias de los datos de la Endes 2017. Esta encuesta fue transversal y se desarrolló con un muestreo de dos etapas, lo que proporciona una inferencia a nivel nacional y regional. Los datos fueron obtenidos desde la plataforma web del INEI en la siguiente dirección: http://iinei.inei.gob.pe/microdatos/. Las bases de datos de la Endes fueron gestionados y analizados mediante el softwareStata/MPversión 16.

La población de estudio estuvo conformada por las madres que participaron en la Endes 2017. Se incluyó a madres de infantes menores de 6 meses que completaron la encuesta del hogar y de la mujer en la Endes 2017, y se excluyó a madres de infantes que habían fallecido antes de aplicar la encuesta.

Los datos fueron analizados considerando el diseño muestral de la Endes, por lo que se utilizó la ponderación muestral de la mujer. En el análisis multivariado, inicialmente se empleó un modelo de regresión de Poisson simple (crudo) para identificar las variables que se incluirán en el modelo de regresión de Poisson múltiple (ajustado); se estimaron las razones de prevalencia, los intervalos de confianza y el nivel de significancia, tomando en cuenta el diseño de la muestra.

\section{DISCUSIÓN}

En la Endes 2017 participaron 21528 madres de infantes menores de 5 años; de ellas, 1828 cumplían los criterios de elegibilidad. La prevalencia de lactancia materna exclusiva a nivel nacional fue del $63,82 \%$ en infantes menores de 6 meses. El 71,21\% de la población de estudio vivía en áreas urbanas, mientras que el 28,79\% lo hacía en áreas rurales. El $48,78 \%$ de las madres alcanzó un nivel educativo de secundaria completa, seguido del 18,19\% que alcanzó un nivel educativo de primaria completa.
Solo el 1,05\% de las madres de infantes menores de 6 meses alcanzaron un nivel de estudios de posgrado.

Según el Índice de riqueza, el 25,55\% de las madres pertenecían al quintil 1 (ingresos económicos bajos); el 26,31\%, al quintil 2 (ingresos económicos bajo-intermedio), y solo el 14,8, al quintil 5 (con ingresos económicos más altos). Respecto de las prácticas que influyen en la salud infantil, el contacto piel a piel oportuno fue practicado por $49,56 \%$ de las madres; asimismo, el 77,9\% de ellas inició su control prenatal durante el primer trimestre de gestación.

En cuanto al acceso a la salud, el 84,05\% conocía cómo acceder a un servicio de salud para recibir atención en caso de emergencia, mientras que el $15,95 \%$ consideraba como un problema el acceso a servicios de salud.

Los departamentos del Perú con mayor prevalencia de práctica de lactancia materna exclusiva durante los seis primeros meses de vida de los infantes fueron Puno (86,34\%), Apurímac (79,44\%), Junín $(78,89 \%)$ y Ayacucho (78,75\%), mientras que los departamentos con menores prevalencias fueron Ica (40,91\%), Tumbes (38,54\%) y Madre de Dios (36,6\%).

Se realizó el análisis bivariado mediante la prueba estadística de chi cuadrado, en la que se identificó que las variables de área de residencia, nivel educativo de la madre, tipo de parto, sexo del infante, índice de riqueza y región natural tuvieron una asociación estadísticamente significativa con la lactancia materna exclusiva en infantes menores de 6 meses $(\mathrm{p}<0,05)$.

Sin embargo, no se encontró asociación estadísticamente significativa con las variables de capacitación en lactancia materna, planificación del embarazo, contacto piel a piel y control prenatal durante el primer trimestre $(p>0,05)$.

Al estimar las medidas de asociación mediante modelos de regresión de Poisson simple, se identificaron las medidas de asociación significativas con las variables de área de residencia, tipo de parto, inicio del control prenatal durante el primer trimestre de gestación, nivel educativo de la madre, índice de riqueza, región natural de residencia y sexo del infante. Estas mostraron significancia estadística mediante sus intervalos de confianza y p-valor. 
Tabla 1. Características de las madres de infantes menores de 6 meses elegibles que participaron en la Endes 2017

\begin{tabular}{|c|c|c|}
\hline Variables & $\begin{array}{l}\text { Frecuencia } \\
(n=1828)\end{array}$ & $\begin{array}{c}\text { Proporciones } \\
\text { ponderadas } \\
(\%)\end{array}$ \\
\hline \multicolumn{3}{|l|}{ Lactancia materna exclusiva } \\
\hline Sí & 1166 & 63,82 \\
\hline No & 662 & 36,18 \\
\hline \multicolumn{3}{|l|}{ Área de residencia } \\
\hline Urbana & 1265 & 71,21 \\
\hline Rural & 563 & 28,79 \\
\hline \multicolumn{3}{|l|}{ Nivel educativo de la madre } \\
\hline Inicial o sin educación & 32 & 1,53 \\
\hline Primaria & 358 & 18,19 \\
\hline Secundaria & 882 & 48,78 \\
\hline Superior no universitario & 290 & 16,86 \\
\hline Superior universitario & 248 & 13,58 \\
\hline Posgrado & 18 & 1,05 \\
\hline \multicolumn{3}{|l|}{ Tipo de parto } \\
\hline Natural & 1217 & 61,22 \\
\hline Cesárea & 611 & 38,78 \\
\hline \multicolumn{3}{|l|}{ Planificación del embarazo } \\
\hline No & 1021 & 55,95 \\
\hline Sí & 807 & 44,05 \\
\hline \multicolumn{3}{|l|}{ Sexo del infante } \\
\hline Masculino & 934 & 51,87 \\
\hline Femenino & 894 & 48,13 \\
\hline \multicolumn{3}{|c|}{ Recibió capacitación en lactancia } \\
\hline No & 673 & 39,77 \\
\hline Sí & 1155 & 60,23 \\
\hline \multicolumn{3}{|l|}{ Índice de riqueza } \\
\hline Quintil 1 & 531 & 25,55 \\
\hline Quintil 2 & 525 & 26,31 \\
\hline Quintil 3 & 341 & 18,17 \\
\hline Quintil 4 & 261 & 15,16 \\
\hline Quintil 5 & 170 & 14,8 \\
\hline \multicolumn{3}{|l|}{ Región natural } \\
\hline Lima Metropolitana & 201 & 28,58 \\
\hline Resto de la Costa & 555 & 26,84 \\
\hline Sierra & 536 & 25,13 \\
\hline Selva & 536 & 19,46 \\
\hline \multicolumn{3}{|l|}{ Contacto piel a piel } \\
\hline No & 831 & 50,44 \\
\hline Sí & 982 & 49,56 \\
\hline \multicolumn{3}{|c|}{ Conoce cómo acceder a un servicio de salud } \\
\hline Sí & 1559 & 84,05 \\
\hline No & 269 & 15,95 \\
\hline
\end{tabular}

\begin{tabular}{lll}
\hline Edad del niño & & \\
\hline 0 meses & 181 & 8,95 \\
\hline 1 mes & 240 & 13,16 \\
\hline 2 meses & 343 & 18,28 \\
\hline 3 meses & 353 & 20,08 \\
\hline 4 meses & 334 & 18,19 \\
\hline 5 meses & 377 & 21,34 \\
\hline Control prenatal durante el primer trimestre & & \\
\hline No & 406 & 22,1 \\
\hline Sí & 1371 & 77,9 \\
\hline
\end{tabular}

Proporciones ponderadas: Se estimaron las proporciones considerando el diseño de la muestra. / Fuente: Elaboración propia

No se identificaron medidas de asociación significativas con la planificación del embarazo, la capacitación en lactancia, el contacto piel a piel ni el conocimiento sobre acceso a los servicios de salud.

Las variables que mostraron significancia estadística enlos modelos de regresión de Poisson simple fueron incluidas en un solo modelo ajustado para estimar sus medidas de efecto y niveles de significancia. En el análisis multivariado, se identificó que las madres de infantes menores de 6 meses de sexo femenino tuvieron un $14 \%$ más de probabilidades de practicar lactancia materna exclusiva que las madres de infantes menores de 6 meses de sexo masculino, lo cual es estadísticamente significativo $(\mathrm{p}=0,006)$ al ajustar por área de residencia, nivel educativo de la madre, tipo de parto, índice de riqueza, región natural de residencia e inicio del control prenatal.

Por otro lado, las madres de infantes menores de 6 meses con ingresos económicos intermedios (quintil 3), según el índice de riqueza, tuvieron un $17 \%$ menos de probabilidades de practicar lactancia materna exclusiva que las madres con ingresos económicos bajos (quintil 1). Esto es estadísticamente significativo $(\mathrm{p}=0,03)$ al ajustar por área de residencia, nivel educativo de la madre, tipo de parto, sexo del infante, región natural de residencia e inicio del control prenatal. Asimismo, las madres de infantes menores de 6 meses con ingresos económicos intermedios-altos (quintil 4), según el índice de riqueza, tuvieron un $20,6 \%$ menos de probabilidades de practicar lactancia materna exclusiva que las madres con ingresos económicos bajos (quintil 1). Esto es estadísticamente significativo $(\mathrm{p}=0,032)$ al ajustar 
Tabla 2. Prevalencia de la práctica de lactancia materna exclusiva de menores de 6 meses de edad, según los departamentos del Perú

\begin{tabular}{|c|c|c|c|c|}
\hline \multirow{3}{*}{ Departamento } & \multicolumn{4}{|c|}{ Lactancia materna exclusiva (LME) } \\
\hline & \multicolumn{2}{|c|}{ No practica LME } & \multicolumn{2}{|c|}{ Sí practica LME } \\
\hline & Frecuencia $(n=662)$ & Proporciones ponderadas (\%) & Frecuencia $(n=1166)$ & Proporciones ponderadas (\%) \\
\hline Amazonas & 20 & 24,51 & 53 & 75,49 \\
\hline Ancash & 12 & 23,5 & 41 & 76,5 \\
\hline Apurímac & 13 & 20,56 & 50 & 79,44 \\
\hline Arequipa & 18 & 31,85 & 39 & 68,15 \\
\hline Ayacucho & 16 & 21,25 & 56 & 78,75 \\
\hline Cajamarca & 20 & 35,9 & 33 & 64,1 \\
\hline Callao & 32 & 43,21 & 44 & 56,79 \\
\hline Cusco & 15 & 25,11 & 47 & 74,89 \\
\hline Huancavelica & 13 & 22,99 & 41 & 77,01 \\
\hline Huánuco & 19 & 28,08 & 55 & 71,92 \\
\hline Ica & 45 & 59 & 31 & 40,91 \\
\hline Junín & 8 & 21,11 & 31 & 78,89 \\
\hline La Libertad & 23 & 33,03 & 44 & 66,97 \\
\hline Lambayeque & 31 & 35,58 & 51 & 64,42 \\
\hline Lima & 78 & 42,19 & 121 & 57,81 \\
\hline Loreto & 32 & 32,14 & 70 & 67,86 \\
\hline Madre de Dios & 52 & 63,4 & 31 & 36,6 \\
\hline Moquegua & 21 & 47,16 & 23 & 52,84 \\
\hline Pasco & 23 & 32,25 & 41 & 67,75 \\
\hline Piura & 28 & 42,72 & 40 & 57,28 \\
\hline Puno & 6 & 13,66 & 32 & 86,34 \\
\hline San Martín & 36 & 41,27 & 45 & 58,73 \\
\hline Tacna & 21 & 35,02 & 40 & 64,98 \\
\hline Tumbes & 50 & 61,46 & 33 & 38,54 \\
\hline Ucayali & 30 & 28,47 & 74 & 71,53 \\
\hline
\end{tabular}

por área de residencia, nivel educativo de la madre, tipo de parto, sexo del infante, región natural de residencia e inicio del control prenatal.

Las madres de infantes menores de 6 meses con ingresos económicos altos (quintil 5), según el índice de riqueza, tuvieron un $29,4 \%$ menos de probabilidades de practicar la lactancia materna exclusiva que las madres con ingresos económicos bajos (quintil 1). Esto es estadísticamente significativo $(\mathrm{p}=0,013)$ al ajustar por área de residencia, nivel educativo de la madre, tipo de parto, sexo del infante, región natural de residencia e inicio del control prenatal.

Las variables como área de residencia, nivel educativo de la madre, tipo de parto, planificación del embarazo, capacitación en lactancia materna, región natural de residencia, contacto piel a piel, inicio del control prenatal y conocimiento sobre el acceso a la salud no se asociaron con la práctica de lactancia materna exclusiva, según el modelo ajustado.

El presente estudio tuvo como objetivo identificar los factores asociados con la lactancia materna exclusiva en el Perú durante el año 2017. Se determinó una prevalencia de práctica de lactancia materna exclusiva del $63,82 \%$ en infantes menores de 6 meses, esto concuerda con las tendencias de lactancia materna del Perú. Al realizar la estimación de la prevalencia de lactancia materna exclusiva, se consideró el diseño muestral de la Endes 2017 y se 
Tabla 3. Análisis bivariado de los factores sociodemográficos y sanitarios con la prevalencia de lactancia materna exclusiva en infantes menores de 6 meses en el Perú

\begin{tabular}{|c|c|c|c|c|c|}
\hline \multirow{3}{*}{ Variables } & \multicolumn{4}{|c|}{ Lactancia materna exclusiva (LME) } & \multirow{3}{*}{ p-valor* } \\
\hline & \multicolumn{2}{|c|}{ No practica LME } & \multicolumn{2}{|c|}{ Sí practica LME } & \\
\hline & $\begin{array}{c}\text { Frecuencia } \\
(n=662)\end{array}$ & $\begin{array}{c}\text { Proporciones } \\
\text { ponderadas (\%) }\end{array}$ & $\begin{array}{l}\text { Frecuencia } \\
(\mathrm{n}=1166)\end{array}$ & $\begin{array}{c}\text { Proporciones } \\
\text { ponderadas (\%) }\end{array}$ & \\
\hline \multicolumn{6}{|l|}{ Área de residencia } \\
\hline Urbana & 521 & 29,1 & 744 & 42,1 & \multirow{2}{*}{$<0,001$} \\
\hline Rural & 141 & 7,07 & 422 & 21,72 & \\
\hline \multicolumn{6}{|l|}{ Nivel educativo de la madre } \\
\hline Inicial o sin educación & 4 & 0,25 & 28 & 1,28 & \multirow{6}{*}{0,006} \\
\hline Primaria & 99 & 4,82 & 259 & 13,36 & \\
\hline Secundaria & 307 & 17,55 & 575 & 31,23 & \\
\hline Superior no universitario & 126 & 6,16 & 164 & 10,25 & \\
\hline Superior universitario & 118 & 6,43 & 130 & 7,14 & \\
\hline Posgrado & 8 & 0,5 & 10 & 0,55 & \\
\hline \multicolumn{6}{|l|}{ Tipo de parto } \\
\hline Natural & 391 & 18,98 & 826 & 42,25 & \multirow{2}{*}{$<0,001$} \\
\hline Cesárea & 271 & 17,2 & 340 & 21,58 & \\
\hline \multicolumn{6}{|l|}{ Planificación del embarazo } \\
\hline No & 352 & 19,84 & 669 & 36,11 & \multirow{2}{*}{0,634} \\
\hline Sí & 310 & 16,34 & 497 & 27,71 & \\
\hline \multicolumn{6}{|l|}{ Sexo del infante } \\
\hline Masculino & 365 & 20,9 & 569 & 30,97 & \multirow{2}{*}{0,005} \\
\hline Femenino & 297 & 15,28 & 597 & 32,86 & \\
\hline \multicolumn{6}{|c|}{ Recibió capacitación en lactancia } \\
\hline No & 247 & 13,98 & 426 & 25,79 & \multirow{2}{*}{0,594} \\
\hline Sí & 415 & 22,2 & 740 & 38,04 & \\
\hline \multicolumn{6}{|l|}{ Índice de riqueza } \\
\hline Quintil 1 & 122 & 5,88 & 409 & 19,67 & \multirow{5}{*}{$<0,001$} \\
\hline Quintil 2 & 186 & 8,34 & 339 & 17,97 & \\
\hline Quintil 3 & 144 & 7,45 & 197 & 10,73 & \\
\hline Quintil 4 & 130 & 6,98 & 131 & 8,18 & \\
\hline Quintil 5 & 80 & 7,53 & 90 & 7,27 & \\
\hline \multicolumn{6}{|l|}{ Región natural } \\
\hline Lima Metropolitana & 87 & 12,54 & 114 & 16,03 & \multirow{4}{*}{$<0,001$} \\
\hline Resto de la Costa & 249 & 11,49 & 306 & 15,35 & \\
\hline Sierra & 128 & 5,85 & 408 & 19,28 & \\
\hline Selva & 198 & 6,29 & 338 & 13,17 & \\
\hline \multicolumn{6}{|l|}{ Contacto piel a piel } \\
\hline No & 321 & 19,24 & 510 & 31,2 & \multirow{2}{*}{0,128} \\
\hline Sí & 326 & 16,46 & 656 & 49,56 & \\
\hline \multicolumn{6}{|c|}{ Conoce cómo acceder a un servicio de salud } \\
\hline Sí & 558 & 30,16 & 1001 & 53,89 & 067 \\
\hline No & 104 & 6,02 & 165 & 9,94 & 0,01 \\
\hline Control prenatal durante el & & & & & \\
\hline No & 128 & 6,68 & 278 & 15,41 & 00 \\
\hline Sí & 511 & 28,81 & 860 & 49,1 &, 00 \\
\hline
\end{tabular}

Proporciones ponderadas: Se estimaron las proporciones considerando el diseño de la muestra. / *Se utilizó la prueba de chi cuadrado para estimar la asociación entre los factores sociodemográficos y la prevalencia de lactancia materna exclusiva. / Fuente: Elaboración propia 
Factores asociados con la lactancia materna exclusiva en el Perú: análisis de la Endes 2017

Tabla 4. Factores asociados con la lactancia materna exclusiva en infantes menores de 6 meses en el Perú

\begin{tabular}{|c|c|c|c|c|c|c|c|c|}
\hline \multirow{2}{*}{ Variables } & \multirow{2}{*}{ RP crudo } & \multicolumn{2}{|c|}{ IC $95 \%$} & \multirow{2}{*}{ p-valor } & \multirow{2}{*}{$\begin{array}{c}\mathrm{RP} \\
\text { ajustado }\end{array}$} & \multicolumn{2}{|c|}{ IC $95 \%$} & \multirow{2}{*}{ p-valor } \\
\hline & & Inferior & Superior & & & Inferior & Superior & \\
\hline \multicolumn{9}{|l|}{ Área de residencia } \\
\hline Urbana & Ref. & & & & Ref. & & & \\
\hline Rural & 1,276 & 1,163 & 1,399 & $<0,001$ & 1,02 & 0,91 & 1,143 & 0,735 \\
\hline \multicolumn{9}{|c|}{ Nivel educativo de la madre } \\
\hline Inicial o sin educación & Ref. & & & & Ref. & & & \\
\hline Primaria & 0,875 & 0,705 & 1,086 & 0,225 & 0,919 & 0,735 & 1,15 & 0,461 \\
\hline Secundaria & 0,762 & 0,626 & 0,929 & 0,007 & 0,905 & 0,726 & 1,129 & 0,376 \\
\hline Superior no universitario & 0,724 & 0,578 & 0,906 & 0,005 & 0,953 & 0,742 & 1,224 & 0,708 \\
\hline Superior universitario & 0,626 & 0,483 & 0,812 & $<0,001$ & 0,9 & 0,669 & 1,21 & 0,484 \\
\hline Posgrado & 0,621 & 0,3 & 1,287 & 0,2 & 0,91 & 0,411 & 2,016 & 0,816 \\
\hline \multicolumn{9}{|l|}{ Tipo de parto } \\
\hline Natural & Ref. & & & & Ref. & & & \\
\hline Cesárea & 0,806 & 0,718 & 0,905 & $<0,001$ & 0,933 & 0,836 & 1,041 & 0,213 \\
\hline \multicolumn{9}{|c|}{ Planificación del embarazo } \\
\hline No & Ref. & & & & & & & \\
\hline Sí & 0,975 & 0,877 & 1,084 & 0,635 & & & & \\
\hline \multicolumn{9}{|l|}{ Sexo del infante } \\
\hline Masculino & Ref. & & & & Ref. & & & \\
\hline Femenino & 1,143 & 1,04 & 1,257 & 0,006 & 1,14 & 1,038 & 1,251 & 0,006 \\
\hline \multicolumn{9}{|c|}{ Recibió capacitación en lactancia } \\
\hline No & Ref. & & & & & & & \\
\hline Sí & 0,974 & 0,884 & 1,073 & 0,593 & & & & \\
\hline \multicolumn{9}{|l|}{ Índice de riqueza } \\
\hline Quintil 1 & Ref. & & & & Ref. & & & \\
\hline Quintil 2 & 0,887 & 0,801 & 0,983 & 0,022 & 0,951 & 0,849 & 1,064 & 0,379 \\
\hline Quintil 3 & 0,767 & 0,669 & 0,879 & $<0,001$ & 0,83 & 0,701 & 0,982 & 0,03 \\
\hline Quintil 4 & 0,701 & 0,585 & 0,84 & $<0,001$ & 0,794 & 0,643 & 0,98 & 0,032 \\
\hline Quintil 5 & 0,638 & 0,508 & 0,801 & $<0,001$ & 0,706 & 0,535 & 0,93 & 0,013 \\
\hline \multicolumn{9}{|l|}{ Región natural } \\
\hline Lima Metropolitana & Ref. & & & & Ref. & & & \\
\hline Resto de la Costa & 1,019 & 0,858 & 1,211 & 0,829 & 0,911 & 0,774 & 1,071 & 0,259 \\
\hline Sierra & 1,367 & 1,169 & 1,599 & $<0,001$ & 1,104 & 0,941 & 1,295 & 0,225 \\
\hline Selva & 1,206 & 1,021 & 1,425 & 0,028 & 0,957 & 0,812 & 1,128 & 0,598 \\
\hline \multicolumn{9}{|l|}{ Contacto piel a piel } \\
\hline No & Ref. & & & & & & & \\
\hline Sí & 1,08 & 0,977 & 1,192 & 0,131 & & & & \\
\hline \multicolumn{9}{|c|}{ Conoce cómo acceder a un servicio de salud } \\
\hline Sí & Ref. & & & & & & & \\
\hline No & 0,971 & 0,848 & 1,113 & 0,675 & & & & \\
\hline \multicolumn{9}{|c|}{ Control prenatal en el primer trimestre } \\
\hline No & Ref. & & & & Ref. & & & \\
\hline$\overline{\text { Sí }}$ & 0,903 & 0,817 & 0,999 & 0,049 & 0,977 & 0,883 & 1,081 & 0,654 \\
\hline
\end{tabular}

Ref.: Referencia. / RP: Razón de prevalencias. / Se realizó el ajuste del modelo con todas las variables que tenían un p-valor significativo en el análisis crudo. / Fuente: Elaboración propia 
analizó los meses de lactancia, como la ingesta de alimentos o bebidas que habían consumido un día antes de la aplicación de la encuesta.

También se identificó que el sexo del infante y el índice de riqueza se asociaron con la práctica de lactancia materna exclusiva en madres de infantes menores de 6 meses. La asociación entre la lactancia materna y el sexo del infante han sido analizadas en otras investigaciones con poblaciones de África $\left({ }^{24,25}\right)$ y Asia $\left({ }^{26}\right)$, donde encontraron que existían diferencias significativas en amamantar según el sexo del infante. Asimismo, algunos estudios sugieren que las madres que dejaron de amamantar al infante percibían que la leche materna no satisfacía las demandas nutricionales del infante, por tal motivo, optaban por iniciar la alimentación complementaria antes de los 6 meses.

Estudios en México $\left({ }^{27}\right)$ y Chile $\left({ }^{28}\right)$ identificaron que las madres amamantaban más tiempo a las niñas que a los niños, según Gonzáles-Cossio et al. Se desconoce por qué las madres introducen antes los alimentos en los niños que en las niñas, pero se cree que las madres perciben que los niños tienen mayores necesidades nutricionales que las niñas; esto da luz a una posible teoría de sesgo de género en la lactancia materna. Sin embargo, en países con sesgo social a favor de los hijos varones como la India, se han identificado mayores frecuencias en la práctica de lactancia materna en los hijos varones, donde existen características culturales y socioeconómicas que contribuyen a dicha práctica.

Además, se ha identificado una asociación significativa entre el índice de riqueza según cuartiles con la práctica de lactancia materna exclusiva en madres de infantes menores de 6 meses, lo que concuerda con algunos estudios en poblaciones de Etiopía $\left({ }^{29}\right)$, Estados Unidos $\left({ }^{30}\right)$, Haití $\left({ }^{31}\right)$, Indonesia $\left({ }^{32}\right)$, Ecuador $\left({ }^{33}\right)$, Brasil $\left({ }^{34}\right)$ y México $\left({ }^{35}\right)$, en los que se identificó la asociación entre ambas variables.

Respecto de las diferencias según el índice de riqueza, las madres con mayor poder adquisitivo pueden acceder a sucedáneos de leche materna o suplementos nutricionales para alimentar al infante antes de los 6 meses de vida, mientras que las madres con ingresos bajos identifican como única fuente de alimentación a la lactancia materna, cuando no pueden pagar alimentos suficientes para su familia. Asimismo, una familia con miembros desempleados o sin soporte familiar incrementa su riesgo de seguridad alimentaria; por lo tanto, la madre opta por quedarse en casa para amamantar al infante como alternativa a la compra de alimentos para él $\left({ }^{31}\right)$.

Asimismo, existen reportes que señalan que las personas con mayor nivel de riqueza pueden tener un estilo de vida y actividades laborales que disminuyen la disponibilidad de tiempo para amamantar durante los primeros meses de vida del infante; por ello, optan por introducir alimentos o sucedáneos de leche materna antes de cumplir esa edad $\left({ }^{36}\right)$.

\section{CONCLUSIONES}

La prevalencia de práctica de lactancia materna exclusiva en infantes menores de 6 meses es del $63,82 \%$ en el Perú. La proporción de madres que residen en áreas rurales y desarrollan una lactancia materna exclusiva durante los seis primeros meses es mayor que la de las madres que residen en áreas urbanas. Las madres que tuvieron un tipo de parto natural practicaron la lactancia materna exclusiva con mayor frecuencia que aquellas que tuvieron un parto por cesárea.

Las madres con ingresos económicos bajos tuvieron mayor probabilidad de practicar la lactancia materna exclusiva que las madres con ingresos económicos intermedios, intermedios-altos y altos.

También, se identificó que las madres de niñas tuvieron mayor propensión a practicar la lactancia materna exclusiva que las que tuvieron niños. Al ajustar por variables confusoras, se demostró una significancia estadística.

La práctica de lactancia materna exclusiva en el Perú concuerda con las metas al 2025, propuestas por la Organización Mundial de la Salud; sin embargo, aún existen determinantes sociales que influyen en su práctica y deben ser abordados por la academia y el sistema de salud peruano. Para ello, es importante promover el desarrollo de estudios con enfoques cuantitativos y cualitativos que contribuyan a mejorar la comprensión de la práctica de la lactancia materna exclusiva tanto en áreas urbanas como rurales. 


\section{CONTRIBUCIÓN DE AUTORÍA}

Rodrigo Daga concibió el estudio, redactó el manuscrito y aprobó la versión final del artículo.

\section{POTENCIALES CONFLICTOS DE INTERESES}

El autor declara que no tiene conflictos de intereses.

\section{FINANCIAMIENTO}

El estudio no tuvo financiamiento.

\section{REFERENCIAS BIBLIOGRÁFICAS}

1. Meline-Quiñones V, Rodríguez-Garrido P, ZangoMartín I. Lactancia materna exclusiva y participación en la vida diaria: una perspectiva ocupacional de la maternidad. Cad Bras Ter Ocup. 2020; 28(1): 86110.

2. Kershenobich D. Lactancia materna en México. Salud Pública Méx. 2017; 59: 346-7.

3. Boccolini CS, de Carvalho ML, de Oliveira MIC. Factors associated with exclusive breastfeeding in the first six months of life in Brazil: a systematic review. Rev Saude Publica. 2015; 49. Disponible en: https://doi.org/10.1590/S0034-8910.2015049005971

4. Hamilton WN, Tarasenko YN. Breastfeeding practices in Georgia: Rural-urban comparison and trend analyses based on 2004-2013 PRAMS data. J Rural Health. 2020; 36(1): 17-26.

5. Ventura AK. Associations between Breastfeeding and Maternal Responsiveness: A Systematic Review of the Literature. Adv Nutr. 2017;8(3):495-510.

6. Alebel A, Tesma C, Temesgen B, Ferede A, Kibret GD. Exclusive breastfeeding practice in Ethiopia and its association with antenatal care and institutional delivery: a systematic review and meta-analysis. Int Breastfeed J. 2018; 13: 31.

7. Alebel A, Dejenu G, Mullu G, Abebe N, Gualu T, Eshetie S. Timely initiation of breastfeeding and its association with birth place in Ethiopia: a systematic review and meta-analysis.IntBreastfeedJ.2017;12:44.

8. Peñacoba C, Catala P. Associations between breastfeeding and mother-infant relationships: a systematic review. Breastfeed Med. 2019; 14(9): 61629.
9. Lutter CK, Morrow AL. Protection, Promotion, and support and global trends in breastfeeding. Adv Nutr. 2013; 4(2): 213-9.

10. Victora CG, Bahl R, Barros AJD, França GVA, Horton S, Krasevec J, et al. Breastfeeding in the 21st century: epidemiology, mechanisms, and lifelong effect. Lancet. 2016; 387(10017): 475-90.

11. Chowdhury R, Sinha B, Sankar MJ, Taneja S, Bhandari N, Rollins $\mathrm{N}$, et al. Breastfeeding and maternal health outcomes: a systematic review and meta-analysis. Acta Paediatr. 2015; 104(Suppl 467): 96-113.

12. Brahm P, Valdés V. Beneficios de la lactancia materna y riesgos de no amamantar. Revista Chilena de Pediatría. 2017; 88(1): 7-14.

13. Piwoz EG, Huffman SL. The impact of marketing of breast-milk substitutes on WHO-recommended breastfeeding practices. Food Nutr Bull. 2015; 36(4): 373-86.

14. Cervera-Gasch Á, Mena-Tudela D, León-Larios F, Felip-Galván N, Rochdi-Lahniche S, Andreu-Pejó L, et al. Female employees' perception of breastfeeding support in the workplace, public universities in Spain: A multicentric comparative study. Int J Environ Res Public Health. 2020; 17(17): 6402.

15. Ratnasari D, Paramashanti BA, Hadi H, MNurs AYN, Astiti D, Nurhayati E. Family support and exclusive breastfeeding among Yogyakarta mothers in employment. Asia Pac J Clin Nutr. 2017; 26(Suppl 1): S31-5.

16. Martin-de-Las-Heras S, Velasco C, Luna-delCastillo JD, Khan KS. Breastfeeding avoidance following psychological intimate partner violence during pregnancy: a cohort study and multivariate analysis. BJOG. 2019; 126(6): 778-83. 
17.Instituto Nacional de Estadística e Informática. Información general de la Encuesta Demográfica y de Salud Familiar 2017 [Internet]. [citado 2 de octubre de 2020]. Disponible en: https://bit. ly/2Mpdg3n

18. Verde CV, Medina MDP, Sifuentes VAN. Lactancia materna exclusiva y factores asociados en madres que asisten a establecimientos de salud de Lima centro. Revista de la Facultad de Medicina Humana. 2020; 20(2): 287-94.

19. Maldonado-Gómez W, Chuan-Ibáñez J, GuevaraVásquez G, Gutiérrez C, Sosa-Flores J. Asociación entre lactancia materna exclusiva y dermatitis atópica en un hospital de tercer nivel de atención. Revista Peruana de Medicina Experimental y Salud Pública. 2019; 36(2): 239-46.

20. Quispe Ilanzo MP, Oyola García AE, Navarro Cancino M, Silva Mancilla JA. Características y creencias maternas asociadas al abandono de la lactancia materna exclusiva. Rev Cub Salud Pública. 2015;41. Disponible en: https://bit.ly/2NNzAE9

21.Lee G, Paredes-Olórtegui $M$, Rengifo-Pinedo S, Ambikapathi R, Peñataro-Yori P, Kosek M, et al. Infant feeding practices in the Peruvian Amazon: implications for programs to improve feeding. Rev Panam Salud Píblica. 2014; 36: 150-7.

22. Quispe C, Terukina R. Niveles de glucemia en recién nacidos a término, adecuados para la edad gestacional, alimentados con leche materna exclusiva y no exclusiva. Anales de la Facultad de Medicina. 2007; 68(2): 125-35.

23. Bahl R, Frost C, Kirkwood BR, Edmond K, Martines $\mathrm{J}$, Bhandari $\mathrm{N}$, et al. Infant feeding patterns and risks of death and hospitalization in the first half of infancy: multicentre cohort study. Bull World Health Organ. 2005; 83: 418-26.

24. Salim YM, Stones W. Determinants of exclusive breastfeeding in infants of six months and below in Malawi: a cross sectional study. BMC Pregnancy Childbirth. 2020; 20(1): 472.

25. Nukpezah RN, Nuvor SV, Ninnoni J. Knowledge and practice of exclusive breastfeeding among mothers in the tamale metropolis of Ghana. Reprod Health. 2018; 15(1): 140.

26. Goyal K, Purbiya P, Lal SN, Kaur J, Anthwal P, Puliyel JM. Correlation of infant gender with postpartum maternal and paternal depression and exclusive breastfeeding rates. Breastfeed Med. 2017; 12: 279 82.
27. González-Cossío T, Moreno-Macías H, Rivera JA, Villalpando S, Shamah-Levy T, Monterrubio EA, et al. Breast-feeding practices in Mexico: results from the Second National Nutrition Survey 1999. Salud Pública Mex. 2003; 45(Suppl 4): S477-89.

28. Niño MR, Silva EG, Atalah SE. Factores asociados a la lactancia materna exclusiva. Revista Chilena de Pediatría. 2012; 83: 161-9.

29. Habtewold TD, Mohammed SH, Endalamaw A, Mulugeta H, Dessie G, Berhe DF, et al. Higher educational and economic status are key factors for the timely initiation of breastfeeding in Ethiopia: A review and meta-analysis. Acta Paediatr. 2020; 109(11): 2208-18.

30. Mercier RJ. Identifying risk factors for not breastfeeding: the interaction of race and economic factors: a case for seeking a local perspective. Breastfeed Med. 2018; 13(8): 544-8.

31.Lesorogol C, Bond C, Dulience SJL, Iannotti L. Economic determinants of breastfeeding in Haiti: The effects of poverty, food insecurity, and employment on exclusive breastfeeding in an urban population. Matern Child Nutr. 2018; 14(2): e12524.

32. Titaley CR, Loh PC, Prasetyo S, Ariawan I, Shankar AH. Socio-economic factors and use of maternal health services are associated with delayed initiation and non-exclusive breastfeeding in Indonesia: secondary analysis of Indonesia Demographic and Health Surveys 2002/2003 and 2007. Asia Pac J Clin Nutr. 2014; 23(1): 91-104.

33. Acosta Silva M, De la Rosa Ferrera JM. Causas que determinan la interrupción de la lactancia materna exclusiva en los barrios Santa Cruz y Propicia I en Esmeraldas, Ecuador. Revista Archivo Médico de Camagüey. 2018; 22: 452-67.

34. Henry BA, Nicolau AIO, Américo CF, Ximenes LB, Bernheim RG, Oriá MOB. Factores socioculturales que influyen en la práctica de la lactancia entre mujeres de baja renta en Fortaleza, Ceará, Brasil: una perspectiva a partir del modelo del sol naciente de leininger. Enfermería Global. 2010; (19).

35. Quezada-Salazar CA, Delgado-Becerra A, ArroyoCabrales LM, Díaz-García MA. Prevalencia de lactancia y factores sociodemográficos asociados en madres adolescentes. Boletín Médico del Hospital Infantil de México. 2008; 65: 19-25.

36. MacMillan Uribe AL, Olson BH. Exploring healthy eating and exercise behaviors among low-income breastfeedingmothers. JHumLact.2019;35(1):59-70. 